\title{
Are All Particles Identical?
}

\author{
Sheldon Goldstein, James Taylor; \\ Roderich Tumulka; and Nino Zanghì
}

September 28, 2004

\begin{abstract}
We consider the possibility that all particles in the world are fundamentally identical, i.e., belong to the same species. Different masses, charges, spins, flavors, or colors then merely correspond to different quantum states of the same particle, just as spin-up and spin-down do. The implications of this viewpoint can be best appreciated within Bohmian mechanics, a precise formulation of quantum mechanics with particle trajectories. The implementation of this viewpoint in such a theory leads to trajectories different from those of the usual formulation, and thus to a version of Bohmian mechanics that is inequivalent to, though arguably empirically indistinguishable from, the usual one. The mathematical core of this viewpoint is however rather independent of the detailed dynamical scheme Bohmian mechanics provides, and it amounts to the assertion that the configuration space for $N$ particles, even $N$ "distinguishable particles," is the set of all $N$-point subsets of physical 3-space.

PACS numbers: 03.65.Ta (foundations of quantum mechanics)
\end{abstract}

\section{Introduction and Overview}

It is not a new idea that what appear to be two different species of particles may in fact be two different states of the same species. It is particularly obvious that spin-up and

*Departments of Mathematics, Physics and Philosophy, Hill Center, Rutgers, The State University of New Jersey, 110 Frelinghuysen Road, Piscataway, NJ 08854-8019, USA. E-mail: oldstein@math.rutgers.edu

${ }^{\dagger}$ Department of Mathematics, Iowa State University, Carver Hall, Ames, IA 50010, USA. E-mail: jostylr@member.ams.org

¥Dipartimento di Fisica dell’Università di Genova and INFN sezione di Genova, Via Dodecaneso 33, 16146 Genova, Italy. E-mail: tumulka@mathematik.uni-muenchen.de

$\S$ Dipartimento di Fisica dell'Università di Genova and INFN sezione di Genova, Via Dodecaneso 33, 16146 Genova, Italy. E-mail: zanghi@ge.infn.it 
spin-down are merely two states of the same particle because we often encounter superpositions, such as spin-left, of these states. But even in cases in which superpositions are extremely hard to obtain, such as of different quark flavors, it is not an unusual idea that what is behind is the same particle, the quark. One may also consider the thought that electrons and positrons are, despite their difference in charge, the same particle; this is suggested in particular by the fact that, while electron states are positive energy solutions of the Dirac equation, positron states are conjugate negative energy solutions of the Dirac equation - a situation similar to left-handed and right-handed photons. Moreover, any explanation of the particular values of masses of the elementary particles would probably have to consist in deriving the appropriate energy eigenvalues for different states - of the same particle. Note also that supersymmetry suggests that the same particle can appear as a boson or a fermion, another example of how one species can appear as two.

We explore here the most extreme possibility of this kind: that all particles are fundamentally identical, i.e., that fundamentally only one species of elementary particles exists. Let us call this the identity hypothesis. This one species would then have to have different states corresponding to being an electron, a quark, a neutrino, or whatever. The consequences of the identity hypothesis are, in a sense, less dramatic than one might expect: we shall point out how every quantum theory involving several particle species can indeed be transformed into a theory of just one species, thus incorporating the identity hypothesis, without any change in the predictions for experiments.

The identity hypothesis can be paraphrased as a statement about the configuration space. The configuration space of a universe of $N$ distinct particles is $\mathbb{R}^{3 N}$, whereas the configuration space of a universe of $N$ identical particles is, as argued in [12, 13, 14, 5, 6], the set of all $N$-element subsets of $\mathbb{R}^{3}$,

$$
{ }^{N} \mathbb{R}^{3}:=\left\{S \subseteq \mathbb{R}^{3} \mid \# S=N\right\} .
$$

This is a manifold of dimension $3 N$ (for a mathematical discussion of this manifold, see [6, 13]). While a configuration in $\mathbb{R}^{3 N}$ indicates that particle 1 is at location $\boldsymbol{Q}_{1} \in \mathbb{R}^{3}$, etc., a configuration from ${ }^{N} \mathbb{R}^{3}$ provides just $N$ points in $\mathbb{R}^{3}$, but no further information on which particle is where. The identity hypothesis amounts to the statement that ${ }^{N} \mathbb{R}^{3}$ should always be considered the natural configuration space, even for a system of $N$ "distinguishable" particles. As we shall show below, an implementation of this idea is provided by a unitary transformation from the standard representation of wave functions as elements of $L^{2}\left(\mathbb{R}^{3 N}, \mathbb{C}^{k_{1}} \otimes \cdots \otimes \mathbb{C}^{k_{N}}\right)$, to a new representation of square-integrable cross-sections of a suitable bundle based on ${ }^{N} \mathbb{R}^{3}$.

To appreciate our proposal, it is helpful to keep in mind that even if a theory treats particles of different species as different sorts of points, associated with different masses, etc., the associated properties are not directly accessible to an observer. When we want to find out whether a given particle is, say, an electron or a muon, we study 
the particle's reaction to various electric fields or other external conditions under our control. For example, when a wavepacket that is a superposition of an electron and a muon is subjected to an electric field, the two contributions to the packet diverge due to their difference in the charge-mass ratio, thus forming two disjoint packets; when the particle is finally detected in the muon packet, we will say it is a muon. Thus, we know that a point in front of us is a muon point rather than an electron point, not because we look into its essence - whatever that would mean - but because its nature is encoded in patterns in its environment. Once one realizes this, the identity hypothesis, which might at first appear to be an outrageous notion, seems like a real possibility.

Within Bohmian mechanics - a precise formulation of quantum mechanics accounting for all quantum phenomena in terms of point particles moving in physical space [4, 8, 10, 9] - this hypothesis acquires an even stronger justification since the theory is primarily about particles, with the wave function having the dynamical role of governing their motion. The choice of ${ }^{N} \mathbb{R}^{3}$ as configuration space corresponds to the insistence that the actual configuration of an $N$-particle system be a set of $N$ points in physical space, with the points labeled in no way, neither by numbers $1, \ldots, N$, nor in the sense that there could be different kinds of points in the world, such as electron points as distinct from muon points or quark points. Given merely an actual configuration set $Q \in{ }^{N} \mathbb{R}^{3}$, there is then simply no fact in the world about what sort of particle there is at a point $\boldsymbol{Q} \in Q$ in physical space, only that there is a particle. This particle is not associated with any label, mass, charge, spin, flavor, or color.

As we shall explain, the identity hypothesis has sharp consequences in the context of Bohmian mechanics: the law of motion has to be suitably adjusted, thus leading to trajectories different from those of the conventional version of the theory. Therefore, the identity hypothesis has a genuine, nontrivial meaning in Bohmian mechanics (and as well in stochastic mechanics [14]). However, arguably, no possible experiment can confirm or disprove the identity hypothesis in the sense of distinguishing between these two versions of Bohmian mechanics. Hence, the identity question remains empirically undecidable - unless one day progress in physics leads to a refined, empirically testable theory of elementary particles that needs the identity hypothesis. However, empirically undecidable does not mean completely undecidable. There may be relevant differences in simplicity and naturalness.

To our knowledge, the consequences of the identity hypothesis have never been discussed in the literature. We are not the first, however, to consider the identity hypothesis. It is implicit in Bell's "Beables for quantum field theory" 2, which has inspired this work. Bell's model, like Bohmian mechanics, involves additional variables beyond the wave function, and as in Bohmian mechanics, these variables are basically particle positions. More precisely, a configuration in Bell's model is described by specifying the number of particles present at every location in (a discrete version of) 3 -space. These 
values are regarded as the actual values of a certain family of observables, given by the fermion number operator at each point in 3-space, which is the sum of the number operators over all (fermion) particle species. Thus, a configuration in Bell's model, like one in ${ }^{N} \mathbb{R}^{3}$, does not distinguish between different kinds of particles.

\section{Standard Bohmian Mechanics}

We begin by describing the conventional version of Bohmian mechanics [4, 10]. For the sake of concreteness, let $\psi: \mathbb{R}^{3 N} \rightarrow \mathbb{C}^{k_{1}} \otimes \cdots \otimes \mathbb{C}^{k_{N}}=\mathbb{W}$ be a quantum mechanical wave function of an $N$-particle system, obeying (as a low-energy description) the Schrödinger equation,

$$
i \hbar \frac{\partial \psi}{\partial t}=-\sum_{i=1}^{N} \frac{\hbar^{2}}{2 m_{i}} \nabla_{i} \cdot \nabla_{i} \psi+V \psi,
$$

where $m_{i}$ denotes the mass of the $i$-th particle, and $V$ the potential which is possibly (Hermitian) matrix valued. The several components of $\psi \in \mathbb{W}$ represent internal degrees of freedom such as spin, flavor, or color. In the usual version of Bohmian mechanics, the $i$-th particle moves according to

$$
\frac{d \boldsymbol{Q}_{i}}{d t}=\frac{\boldsymbol{j}_{i}(Q)}{\rho(Q)}
$$

where $Q=\left(\boldsymbol{Q}_{1}, \ldots, \boldsymbol{Q}_{N}\right)$ is the configuration in $\mathbb{R}^{3 N}$,

$$
\rho=\psi^{*} \psi
$$

(meaning a scalar product in $\mathbb{W}$ ) is the probability density, and

$$
\boldsymbol{j}_{i}=\frac{\hbar}{m_{i}} \operatorname{Im} \psi^{*} \nabla_{i} \psi
$$

is the probability current, more precisely the part of it corresponding to the $i$-th particle.

We have that if $Q(0)$ is random with distribution $\rho_{0}$ (as we shall assume in the following), then $Q(t)$ has distribution $\rho_{t}$ for every time $t$. This follows from the fact that $\rho$ and the velocity field $v$ on $\mathbb{R}^{3 N}$ defined in (3) and (4) obey, by virtue of the Schrödinger equation (2), a continuity equation

$$
\frac{\partial \rho}{\partial t}=-\operatorname{div}(\rho v)
$$

on $\mathbb{R}^{3 N}$. This is an extremely important property, as it expresses a certain compatibility between the two equations of motion defining the dynamics, which we call the equivariance of $\rho=|\psi|^{2}$ [8]. Such a notion plays a crucial role in establishing the empirical import of the theory [4, 8, 9]. 


\section{Identity-based Bohmian Mechanics}

Bohmian versions of quantum theory define a dynamics on some configuration space $\mathcal{Q}$; the choice $\mathcal{Q}={ }^{N} \mathbb{R}^{3}$ reflects the identity hypothesis, as the particles themselves are then not associated with labels, masses, charges, etc. - which are implicit, though, in the wave function, and relevant to its time evolution (2). With such a choice the actual configuration $Q(t)$ would move in ${ }^{N} \mathbb{R}^{3}$, whereas $\mathbb{R}^{3 N}$ would play a role merely for the wave function.

A crucial observation is that a dynamics on ${ }^{N} \mathbb{R}^{3}$ is given by a symmetric (permutation invariant) dynamics on $\mathbb{R}^{3 N}$. However, (3) is not symmetric (and thus does not define a dynamics on ${ }^{N} \mathbb{R}^{3}$ and fails to be compatible with the identity hypothesis), except in the special case that the masses are all the same, the potential $V$ is permutation invariant, and the wave function is symmetric or antisymmetric.

We are thus led to consider the dynamics on $\mathbb{R}^{3 N}$ obtained by replacing (3) from the conventional version with

$$
\frac{d \boldsymbol{Q}_{i}}{d t}=\frac{\sum_{\sigma} \boldsymbol{j}_{\sigma(i)}(\sigma Q)}{\sum_{\sigma} \rho(\sigma Q)}
$$

where the sums are taken over the group $S_{N}$ of permutations of $N$ elements, and $\sigma Q=$ $\left(\boldsymbol{Q}_{\sigma^{-1}(1)}, \ldots, \boldsymbol{Q}_{\sigma^{-1}(N)}\right) .{ }^{1}$ In words, the velocity of (17) is obtained from the one of (3) by symmetrizing the current and the density, i.e., by averaging over all permutations of the $N$ particles. Note that this is different from symmetrizing the wave function, a procedure that would lead to zero in the presence of fermions. Note also that this is different from directly symmetrizing the velocity (3), which would also define a dynamics on ${ }^{N} \mathbb{R}^{3}$, but one for which, unlike with (7), the dynamics obtained will not be equivariant, see below. Since (3) and (7) define different velocities, they lead to different trajectories and thus inequivalent theories.

We emphasize that the theory defined by (71) is permutation invariant in a strong sense, a sense in which the one defined by (3) is not: if $Q(t)$ and $Q^{\prime}(t)$ are two solution curves of (17) in configuration space $\mathbb{R}^{3 N}$, and if at some time $Q^{\prime}=\sigma Q$ for some permutation $\sigma \in S_{N}$, then $Q^{\prime}(t)=\sigma Q(t)$ at all times $t$. By virtue of this permutation invariance, (7) defines a dynamics on ${ }^{N} \mathbb{R}^{3}$ via projection. After all, if we know the set of $N$ points contained in the configuration $Q \in \mathbb{R}^{3 N}$ at some time, then this information is sufficient to determine the configuration $Q(t)$ at all times, modulo the ordering. We call the dynamics on ${ }^{N} \mathbb{R}^{3}$ thus obtained identity-based Bohmian mechanics.

To get a handle on identity-based Bohmian mechanics and its symmetry properties, consider the very simple example of two particles, say an electron (particle 1) and a muon (particle 2), with scalar-valued wave function $\psi\left(q_{1}, q_{2}\right)=\phi\left(q_{1}\right) \chi\left(q_{2}\right)$, which are,

\footnotetext{
${ }^{1}$ This means the particle at $\boldsymbol{Q}_{i}$ gets the number $\sigma(i)$, defining its new place in the ordering. Note that $\boldsymbol{j}_{\sigma(i)}(\sigma Q)$ lies, for every $\sigma$, in the tangent space to $\mathbb{R}^{3}$ at $\boldsymbol{Q}_{i}$, if we wish to distinguish between tangent spaces at different points.
} 
say, evolving according to the free Hamiltonian (so that factorization is preserved). Then, according to (3) , the equations of motion of standard Bohmian mechanics are

$$
\begin{aligned}
\frac{d \boldsymbol{Q}_{1}}{d t} & =\frac{\hbar}{m_{e}} \operatorname{Im} \frac{\nabla \phi\left(\boldsymbol{Q}_{1}\right)}{\phi\left(\boldsymbol{Q}_{1}\right)} \\
\frac{d \boldsymbol{Q}_{2}}{d t} & =\frac{\hbar}{m_{\mu}} \operatorname{Im} \frac{\nabla \chi\left(\boldsymbol{Q}_{2}\right)}{\chi\left(\boldsymbol{Q}_{2}\right)}
\end{aligned}
$$

where $m_{e}$ and $m_{\mu}$ are respectively the masses of the electron and of the muon. On the other hand, according to (7), the dynamics of motion of identity-based Bohmian mechanics is given by

$$
\begin{aligned}
\frac{d \boldsymbol{Q}_{1}}{d t} & =\frac{\frac{\hbar}{m_{e}}\left|\chi\left(\boldsymbol{Q}_{2}\right)\right|^{2} \operatorname{Im}\left[\phi\left(\boldsymbol{Q}_{1}\right)^{*}(\nabla \phi)\left(\boldsymbol{Q}_{1}\right)\right]+\frac{\hbar}{m_{\mu}}\left|\phi\left(\boldsymbol{Q}_{2}\right)\right|^{2} \operatorname{Im}\left[\chi\left(\boldsymbol{Q}_{1}\right)^{*}(\nabla \chi)\left(\boldsymbol{Q}_{1}\right)\right]}{\left|\phi\left(\boldsymbol{Q}_{1}\right)\right|^{2}\left|\chi\left(\boldsymbol{Q}_{2}\right)\right|^{2}+\left|\phi\left(\boldsymbol{Q}_{2}\right)\right|^{2}\left|\chi\left(\boldsymbol{Q}_{1}\right)\right|^{2}} \\
\frac{d \boldsymbol{Q}_{2}}{d t} & =\frac{\frac{\hbar}{m_{\mu}}\left|\phi\left(\boldsymbol{Q}_{1}\right)\right|^{2} \operatorname{Im}\left[\chi\left(\boldsymbol{Q}_{2}\right)^{*}(\nabla \chi)\left(\boldsymbol{Q}_{2}\right)\right]+\frac{\hbar}{m_{e}}\left|\chi\left(\boldsymbol{Q}_{1}\right)\right|^{2} \operatorname{Im}\left[\phi\left(\boldsymbol{Q}_{2}\right)^{*}(\nabla \phi)\left(\boldsymbol{Q}_{2}\right)\right]}{\left|\phi\left(\boldsymbol{Q}_{1}\right)\right|^{2}\left|\chi\left(\boldsymbol{Q}_{2}\right)\right|^{2}+\left|\phi\left(\boldsymbol{Q}_{2}\right)\right|^{2}\left|\chi\left(\boldsymbol{Q}_{1}\right)\right|^{2}}
\end{aligned}
$$

Note that now the two particle indices 1 and 2 do not carry any direct relation to the particle species (as characterized by their masses): Contrary to the velocity formulas (8) and (9), there is nothing in the right hand side of (10) and (11) that distinguishes $\boldsymbol{Q}_{1}$ from $\boldsymbol{Q}_{2}$. The two particles are distinguished, rather, only by the positions they happen to have at any given time. Note in particular that if $\phi$ and $\chi$ have disjoint supports, with, say, $\phi$ supported on "the left" and $\chi$ on "the right," then only one term in the numerator and one in the denominator of (10) and (11) will be nonvanishing. Then $\boldsymbol{Q}_{1}$ will behave like an electron, resp. like a muon, when it is on the left, resp. right. In other words, the particle on the left behaves like an electron and the one on the right like a muon, regardless of how we might label them.

More generally, for arbitrary two-particle wave function and Hamiltonian, consider a configuration with an electron at $\boldsymbol{x} \in \mathbb{R}^{3}$ and a muon at $\boldsymbol{y} \in \mathbb{R}^{3}$, and another configuration with the muon at $\boldsymbol{x}$ and the electron at $\boldsymbol{y}$. In both configurations, (7) yields the same velocity for the particle at $\boldsymbol{x}$, regardless of whether it is the electron or the muon (and the same velocity at $\boldsymbol{y}$ ). With (3), in contrast, there is no reason why this should be so.

The equation of motion for $Q \in{ }^{N} \mathbb{R}^{3}$ can be written as

$$
\frac{d Q}{d t}=v_{t}(Q)
$$

where $v=v_{t}$ is a time-dependent vector field on ${ }^{N} \mathbb{R}^{3}$ that is determined by (7). Similarly, the denominator of (7) defines a probability density $\rho$ on ${ }^{N} \mathbb{R}^{3}$, while its numerator defines a probability current $J=\rho v$ on ${ }^{N} \mathbb{R}^{3}$. 
As in conventional Bohmian mechanics, also in identity-based Bohmian mechanics we have that if $Q(0)$ is chosen at random with distribution $\rho_{0}$, then $Q(t)$ has distribution $\rho_{t}$ for every time $t$. This is a consequence of the fact that $\rho$ and $v$ obey the continuity equation

$$
\frac{\partial \rho}{\partial t}=-\operatorname{div}(\rho v)
$$

on ${ }^{N} \mathbb{R}^{3}$, which follows from (6) by summing both sides over all permutations.

\section{Wave Function on ${ }^{N} \mathbb{R}^{3}$}

If the configuration space is $\mathcal{Q}={ }^{N} \mathbb{R}^{3}$, then one might imagine that the wave function should also live on ${ }^{N} \mathbb{R}^{3}$ rather than on $\mathbb{R}^{3 N}$. And that is possible! One may transform any wave function $\psi: \mathbb{R}^{3 N} \rightarrow \mathbb{W}=\mathbb{C}^{k_{1}} \otimes \cdots \otimes \mathbb{C}^{k_{N}}$ into a cross-section $\phi$ of a suitable vector bundle $E$ over ${ }^{N} \mathbb{R}^{3}$. The fiber space $E_{q}$ at $q \in{ }^{N} \mathbb{R}^{3}$ of this bundle has dimension $N ! k_{1} \cdots k_{N}$ and can be defined as

$$
E_{q}=\bigoplus_{\nu \in B_{q}} \mathbb{W}
$$

where $B_{q}$ is the set of all bijections $q \rightarrow\{1, \ldots, N\}$; thus, $\nu$ runs through all possible numberings, or all possible identifications of the $N$ points with the $N$ particle "identities." In particular, $E_{q}$ is the direct sum of $N$ ! copies of $\mathbb{W}$. (It follows that even for $k_{1}=\cdots=k_{N}=1$ (spinless particles), the wave function $\phi(q)$ has $N$ ! components at $q \in \mathcal{Q}$, each component corresponding to a particular way of labeling the points.) We define the scalar product in $E_{q}$ in such a way that (14) is an orthogonal sum.

Another way of viewing $E_{q}$ is this: every $\nu \in B_{q}$ defines an ordering of the $N$ points of $q$, and thus an element of $\mathbb{R}^{3 N}$, namely $\hat{q}=\left(\nu^{-1}(1), \ldots, \nu^{-1}(N)\right)$. We shall sometimes write $(q, \nu)$ for $\hat{q} . E_{q}$ is the direct sum of the value spaces (each being a copy of $\mathbb{W}$ ) of $\psi$ at the points $\hat{q} \in \mathbb{R}^{3 N}$. (This view of $E_{q}$ allows a generalization to the case that $\psi$ is itself a cross-section of a vector bundle over $\mathbb{R}^{3 N}$.) And this view is, in fact, how the transformation of $\psi$ into $\phi$ can best be understood:

$$
\phi(q)=\oplus_{\nu} \psi(q, \nu)=\oplus_{\nu} \psi(\hat{q}) .
$$

Conversely, $\psi$ can be reconstructed from $\phi$ at all configurations $\left(\boldsymbol{q}_{1}, \ldots, \boldsymbol{q}_{N}\right)$ without coincidences (i.e., such that $\boldsymbol{q}_{i} \neq \boldsymbol{q}_{j}$ whenever $i \neq j$ ):

$$
\psi\left(\boldsymbol{q}_{1}, \ldots, \boldsymbol{q}_{N}\right)=\phi_{\nu}\left(\left\{\boldsymbol{q}_{1}, \ldots, \boldsymbol{q}_{N}\right\}\right)
$$

with $\nu\left(\boldsymbol{q}_{i}\right):=i$. Since the configurations with coincidences form a null set in $\mathbb{R}^{3 N}$, the transformation $\psi \mapsto \phi$ defines a unitary identification between the Hilbert spaces 
$L^{2}\left(\mathbb{R}^{3 N}, \mathbb{W}\right) \rightarrow L^{2}(E)$, where by $L^{2}(E)$ we denote the space of square-integrable crosssections of the bundle $E$.

(We note in passing that in identity-based Bohmian mechanics the notion of conditional wave function for subsystems [8 is defined much less often than in standard Bohmian mechanics. For example, in standard Bohmian mechanics the conditional wave function for scalar-valued wave functions is always defined, but this is not the case in identity-based Bohmian mechanics, due to the fact that the "particle identities" are then internal degrees of freedom (see above), more or less like spin in standard Bohmian mechanics. Thus in identity-based Bohmian mechanics only the notion of conditional density matrix is always well defined [7. This may appear paradoxical, in view of our claim that the two theories are empirically equivalent (see below). There is, however, no paradox: upon reflection we realize that whenever we would know, in a standard Bohmian universe, that the wave function of a subsystem is $\psi$, then, for identity based Bohmian mechanics, its conditional density matrix would in fact be $|\psi\rangle\langle\psi|$.)

\section{Dynamics in Terms of $\phi$}

For the sake of completeness, we explicitly describe in this section how the identitybased Bohmian theory can be formulated purely in terms of the bundle cross-section $\phi$. First note that

$$
|\phi(q)|^{2}=\sum_{\nu \in B_{q}}|\psi(q, \nu)|^{2}
$$

so that $\rho(q)=|\phi(q)|^{2}$. A connection (covariant derivative operator) $\nabla$ can be defined on $E$ in an obvious way. ${ }^{2}$ The component of the probability current relative to the particle at $\boldsymbol{q} \in q$ is given by

$$
\boldsymbol{j}_{\boldsymbol{q}}(q)=\sum_{\nu} \frac{\hbar}{m_{\nu(\boldsymbol{q})}} \operatorname{Im} \phi_{\nu}^{*}(q) \nabla_{\boldsymbol{q}} \phi_{\nu}(q),
$$

and we obtain the following formula for the vector field $v$ in terms of $\phi$ :

$$
\boldsymbol{v}_{\boldsymbol{q}}=\frac{\boldsymbol{j}_{\boldsymbol{q}}}{\rho}
$$

where $\boldsymbol{v}_{\boldsymbol{q}}$ is the component of $v(q)$ corresponding to $\boldsymbol{q} \in q$. In another notation,

$$
\frac{d \boldsymbol{Q}}{d t}=\sum_{\nu} \frac{\hbar}{m_{\nu(\boldsymbol{Q})}} \operatorname{Im} \frac{\phi_{\nu}^{*}(Q) \nabla_{\boldsymbol{Q}} \phi_{\nu}(Q)}{\phi^{*}(Q) \phi(Q)} .
$$

\footnotetext{
${ }^{2}$ Here is the definition, in terms of parallel transport: if $q(s), 0 \leq s \leq 1$, is a curve in ${ }^{N} \mathbb{R}^{3}$ then any bijection $\nu_{0}: q(0) \rightarrow\{1, \ldots, N\}$ can be transported along the curve by having the numbering follow the points of $q(s)$ as they move continuously from $q(0)$ to $q(1)$, thus defining a final bijection $\nu_{1}: q(1) \rightarrow\{1, \ldots, N\}$. Parallel transport of some element $e(0) \in E_{q(0)}$ along the curve leads to $\oplus_{\nu_{1}} e_{\nu_{0}}(0)=: e(1) \in E_{q(1)}$. This connection is nontrivial (i.e., parallel transport along a loop may differ from the identity) but flat (i.e., has curvature zero).
} 
The time evolution of $\phi$ obeys

$$
i \hbar \frac{\partial \phi_{\nu}}{\partial t}(q)=-\sum_{\boldsymbol{q} \in q} \frac{\hbar^{2}}{2 m_{\nu(\boldsymbol{q})}} \nabla_{\boldsymbol{q}} \cdot \nabla_{\boldsymbol{q}} \phi_{\nu}(q)+V(q, \nu) \phi_{\nu}(q) .
$$

\section{A Remark on Identical Particles}

It is interesting to see what happens when we apply the transformation (15) to a system of identical particles, i.e., of bosons or fermions. If $\psi$, with value space $\mathbb{W}=\left(\mathbb{C}^{k}\right)^{\otimes N}$, represents a system of $N$ bosons, then it must be symmetric under permutation. As a consequence, $\psi(q, \nu)=R_{\nu^{\prime} \circ \nu^{-1}} \psi\left(q, \nu^{\prime}\right)$, where for any permutation $\sigma \in S_{N}$ of the particles, $R_{\sigma}:\left(\mathbb{C}^{k}\right)^{\otimes N} \rightarrow\left(\mathbb{C}^{k}\right)^{\otimes N}$ is the linear mapping that correspondingly permutes the components of $\psi$. It follows that

$$
\phi_{\nu}=R_{\nu^{\prime} \circ \nu^{-1}} \phi_{\nu^{\prime}}
$$

so that $\phi$ is actually confined to a subbundle of $E$, characterized by (19) and having dimension $k^{N}$ rather than $N ! k^{N}$. The subbundle is parallel with respect to the connection, i.e., parallel transport will remain within the subbundle. For $k=1, R_{\sigma}$ is the identity, and the subbundle defined by (19) is the trivial bundle $\mathcal{Q} \times \mathbb{C}$, so that $\phi$ can be identified with a function ${ }^{N} \mathbb{R}^{3} \rightarrow \mathbb{C}$.

Now consider a system of fermions. Then $\psi$ is anti-symmetric under permutations, so that $\psi(q, \nu)=(-1)^{\nu^{\prime} \circ \nu^{-1}} R_{\nu^{\prime} \circ \nu^{-1}} \psi\left(q, \nu^{\prime}\right)$ where $(-1)^{\sigma}$ denotes the sign of the permutation $\sigma$, and consequently,

$$
\phi_{\nu}=(-1)^{\nu^{\prime} \circ \nu^{-1}} R_{\nu^{\prime} \circ \nu^{-1}} \phi_{\nu^{\prime}}
$$

In this case, $\phi$ is confined to another parallel subbundle of $E$, characterized by (20) and also having dimension $k^{N}$. For $k=1$, this subbundle can be called the Fermi line bundle; it has been described in 13, 15, 6. The connection is such that parallel transport along a closed curve in ${ }^{N} \mathbb{R}^{3}$ that realizes a permutation $\sigma$ coincides with multiplication by $(-1)^{\sigma}$, the sign of $\sigma$.

Finally, when the system of $N$ particles under consideration contains $N_{1}$ identical particles of species $1, \ldots$, and $N_{\ell}$ identical particles of species $\ell$, so that $N_{1}+\ldots+$ $N_{\ell}=N$, then $\phi$ is confined to a subbundle $F$ of dimension $\left(N ! / N_{1} ! \cdots N_{\ell} !\right) k_{1}^{N_{1}} \cdots k_{\ell}^{N_{\ell}}$, characterized by one condition like either (19) or (20) for each species.

\section{Another Approach to Identity-Based Bohmian Me- chanics}

In this section we briefly describe a different perspective on identity-based Bohmian mechanics that lies outside the main line of this paper and in particular is not essential 
for an understanding of our main points. We have, in the previous sections, formulated identity-based Bohmian mechanics on ${ }^{N} \mathbb{R}^{3}$, starting from conventional Bohmian mechanics on $\mathbb{R}^{3 N}$. This brings us to the question as to whether one could arrive at this

formulation without ever invoking $\mathbb{R}^{3 N}$. We now sketch how to do this for bosons. For a discussion of fermions see [15, Section 5.9].

Suppose that the state space $W$, representing the internal degrees of freedom for a single particle, is the sum of the state spaces $W_{j}$ of $\ell$ different species,

$$
W=\bigoplus_{j=1}^{\ell} W_{j}, \quad W_{j}=\mathbb{C}^{k_{j}}
$$

This means that the particle can be in a superposition of being an electron, a muon, etc. For $N$ bosons with state space $W$, the wave function $\phi$ is a cross-section of the bundle $E^{\prime}$ defined by

$$
E_{q}^{\prime}=\bigotimes_{q \in q} W
$$

see [6] for a detailed discussion. The requirement that we have $N_{1}$ particles of species $1, \ldots$, and $N_{\ell}$ particles of species $\ell$ defines a subbundle $F^{\prime}$ of $E^{\prime}$ which can be identified with the bundle $F$ introduced in the last paragraph of Section [6, in the case of bosons. A cross-section of $F^{\prime}$ remains in $F^{\prime}$ under the Schrödinger evolution, and the obvious Bohmian dynamics on ${ }^{N} \mathbb{R}^{3}$ associated with cross-sections of $E^{\prime}$ (see [15, 6] for the explicit definition) agrees for cross-sections of $F^{\prime}$ with the dynamics defined by (17) for the corresponding cross-section of $F$.

\section{Empirical Equivalence}

One may wonder whether identity-based and conventional Bohmian mechanics are empirically distinguishable, i.e., whether any possible experiment could enable us to decide between these two versions of Bohmian mechanics. The question is delicate since it relates to the meaning of empirical equivalence in general; we touch a bit more on this issue in a separate work [11]. That the answer arguably is no can however be appreciated quite easily by reflecting upon the three following points:

- The outcomes of all conceivable experiments will be recorded in the unordered configuration $\left\{\boldsymbol{Q}_{1}, \ldots, \boldsymbol{Q}_{N}\right\}$. To illustrate this fact, we may imagine the outcome as given by the orientation of a pointer on a scale; as the pointer consists of a huge number of electrons and quarks, for reading off the orientation of the pointer we need not be explicitly told which points are the electrons and which are the quarks. 
- The label of a particle in a standard Bohmian universe is not directly perceptible to an observer. We would base the decision whether, say, a given particle is an electron or a muon on how the particle moves under certain conditions that we control, say in terms of wave packets that spatially diverge due to differences (as the charge-mass ratio) encoded in the wave function ${ }^{3}$ and finally grounded (after the experiment has been completed) in macroscopic patterns in the environment. This fact strongly suggests that only $\left\{\boldsymbol{Q}_{1}, \ldots, \boldsymbol{Q}_{N}\right\}$ and not $\left(\boldsymbol{Q}_{1}, \ldots, \boldsymbol{Q}_{N}\right)$ could ultimately be empirically relevant.

- In conventional Bohmian mechanics, the distribution of the configuration $Q$ is $|\psi|^{2}$ at any time (see the end of Section 21). ${ }^{4}$ If we ignore the labeling of the particles, we obtain from $Q=\left(\boldsymbol{Q}_{1}, \ldots, \boldsymbol{Q}_{N}\right)$ the set $\left\{\boldsymbol{Q}_{1}, \ldots, \boldsymbol{Q}_{N}\right\} \in{ }^{N} \mathbb{R}^{3}$ whose (marginal) distribution coincides with the distribution $\rho$ of the configuration of identity-based Bohmian mechanics (see the end of Section 31). Since any empirical decision, if it can be made at some time (after, say, an experiment has been performed), must be based on the configuration (of systems, apparatuses, and the rest of the universe) at that time, two theories predicting the same distribution for the configuration $\left\{\boldsymbol{Q}_{1}, \ldots, \boldsymbol{Q}_{N}\right\}$ cannot presumably be distinguished by experiment.

To sum up, it seems that no experiment could enable us to decide between conventional and identity-based Bohmian mechanics. It is presumably also true of orthodox quantum mechanics that no experiment could enable us to distinguish an identity-based version from the conventional version; this conclusion is based on the special status of position measurements - if only the positions of instrument pointers - a fact whose relevance has been stressed with great force by Bell [3].

\section{References}

[1] Bassi, A., Ghirardi, G.C.: "Dynamical Reduction Models," Phys. Rep. 379, 257427 (2003), quant-ph/0302164.

[2] Bell, J. S.: "Beables for quantum field theory," Phys. Rep. 137, 49-54 (1986). Reprinted in Bell, J. S.: Speakable and unspeakable in quantum mechanics. Cambridge: Cambridge University Press (1987), p. 173.

\footnotetext{
${ }^{3}$ For an elementary illustration of this fact, recall the example discussed in Section 3 as remarked there, when the supports of $\phi$ and $\chi$ are disjoint, (10) and (11) reduce to (18) and (9), up to a possible permutation of the indices 1 and 2 .

${ }^{4}$ In the case that empirical evidence contradicting quantum mechanics should be found, such as, e.g., evidence for spontaneous wave function collapse [1] or for violations of the quantum equilibrium distribution $|\psi|^{2}$ (as studied particularly by A. Valentini [16]), both conventional and identity-based Bohmian mechanics [understood as (quantum) equilibrium theories], though still empirically equivalent to each other, would become empirically inadequate: their predictions would be wrong.
} 
[3] Bell, J. S.: "On the Impossible Pilot Wave," Found. Phys. 12, 989-999 (1982). Reprinted in Bell, J. S.: Speakable and unspeakable in quantum mechanics. Cambridge: Cambridge University Press (1987), p. 159.

[4] Bohm, D.: "A Suggested Interpretation of the Quantum Theory in Terms of "Hidden" Variables. I," Phys. Rev. 85, 166-179 (1952). Bohm, D.: "A Suggested Interpretation of the Quantum Theory in Terms of "Hidden" Variables. II," Phys. Rev. 85, 180-193 (1952).

[5] Brown, H., Sjöqvist, E., Bacciagaluppi, G.: "Remarks on identical particles in de Broglie-Bohm theory," Phys. Lett. A 251, 229-235 (1999), quant-ph/9811054.

[6] Dürr, D., Goldstein, S., Taylor, J., Tumulka, R., Zanghì, N.: "Bosons, Fermions, and the Topology of Configuration Space," to be submitted.

[7] Dürr, D., Goldstein, S., Tumulka, R., Zanghì, N.: "On the Role of Density Matrices in Bohmian Mechanics," to appear in Found. Phys., quant-ph/0311127.

[8] Dürr, D., Goldstein, S., Zanghì, N.: "Quantum Equilibrium and the Origin of Absolute Uncertainty," J. Stat. Phys. 67, 843-907 (1992), quant-ph/0308039.

[9] Dürr, D., Goldstein, S., Zanghì, N.: "Quantum Equilibrium and the Role of Operators as Observables in Quantum Theory," J. Stat. Phys. 116, 959-1055 (2004), quant-ph/0308038.

[10] Goldstein, S.: "Bohmian Mechanics" (2001), in Stanford Encyclopedia of Philosophy, edited by E. N. Zalta, published online by Stanford University, http://plato.stanford.edu/entries/qm-bohm.

[11] Goldstein, S., Taylor, J., Tumulka, R., Zanghì, N.: "Are All Particles Real?" quant-ph/0404134.

[12] Laidlaw, M.G., DeWitt, C.M.: "Feynman functional integrals for systems of indistinguishable particles," Phys. Rev. D 3, 1375-1378 (1971).

[13] Leinaas, J. M., Myrheim, J.: "On the Theory of Identical Particles," Il Nuovo Cimento 37, 1-23 (1977).

[14] Nelson, E.: Quantum Fluctuations. Princeton: Princeton University Press (1985).

[15] Taylor, J.: "Connections with Bohmian Mechanics," Ph. D. Thesis, Department of Mathematics, Rutgers University (2003).

[16] Valentini, A.: "Subquantum Information and Computation," Pramana - J. Phys. 59, 269-277 (2002), quant-ph/0203049. 